\section{Response to Bauer et al.}

To the Editor: I appreciate the opportunity to respond to the letter "Unmet needs in human genomic variant interpretation" by Bauer et al. ${ }^{1}$ It is worth noting that although the letter focuses on my own perspectives, as outlined in my Commentary, ${ }^{2}$ most of the issues raised address the activities of ClinGen and ClinVar and, as such, I have engaged the input of the other leaders of these programs in my responses.

ClinGen, which focuses on the expert curation of genes and variants, is primarily funded through three National Institutes of Health (NIH) grants awarded to nine principal investigators; however, ClinGen's expert panels and working groups involve more than 500 individuals from over 250 academic institutions and companies worldwide, many of whom are volunteers or are supported by their own employers. ClinGen investigators interact regularly with members from around the world and these enriching exchanges have both supported and informed our approaches. We continue to welcome new ideas and collaborations with both academia and industry to ensure that we support the best and most sustainable resources for the global community.

ClinGen has partnered with ClinVar to support its role as the primary site for deposition and retrieval of variant evidence and annotations from individual laboratories. Before choosing this partnership, a group of clinical laboratory directors spent several years evaluating approaches to supporting our needs, also trying another approach $\left(\right.$ MutaDATABASE $^{3}$ ) involving a single commercial partner. However, this endeavor was not successful and we ultimately decided to partner with the National Center for Biotechnology Information, given its ability to provide sustainable funding without commercial influence and its willingness to partner closely with the community to serve its needs, as it did in an early partnership with the International Standards for Cytogenomics Arrays consortium. No clinical laboratory has been forced to submit to ClinVar. One of the first ClinGen grants provided a small amount of funding to six laboratories to pioneer approaches for ClinVar submission; however, $\mathrm{NIH}$ funding is no longer provided to any individual clinical laboratory to fund submissions. Submission is funded by the clinical laboratories themselves, and it is currently their choice where to share their knowledge. The fact that hundreds of clinical laboratories voluntarily submit to ClinVar is objective evidence of the widespread support for ClinVar.

It is important to note that ClinGen fully supports engagement with industry, and many members of our working groups and expert panels are from industry. However, we do not allow any expert panel or working group to have overrepresentation from individual commercial or academic entities to ensure conflicts of interest are managed and consensus across groups can be achieved.

ClinVar is a global database with submissions from over 60 countries (http://www.ncbi.nlm.nih.gov/clinvar/docs/map). ClinVar launched in April 2013 and has processed over 500,000 records from more than 800 submitters, clearly demonstrating its capability to support the sharing needs of the submitters. Substantial work goes into standardizing data into consistent formats for representation in ClinVar. ClinVar is now considering automated submission methods, as well as tools to help laboratories quality control their data in advance of submission, both of which will aid in reducing the time from submission to posting, as well as allowing submissions to scale, if needed, in the future. We have also been working closely with the Leiden Open Variation Database (LOVD) on plans to support the submission of curated variants into ClinVar, allowing groups who wish to maintain their patient data in LOVD to still harmonize their knowledge with the broader community through ClinVar.

While we agree with the authors that interpretation has improved with the American College of Medical Genetics and Genomics-Association for Molecular Pathology guidelines, these guidelines continue to evolve and require gene-level specification. The application of criteria requires judgment, leading laboratories to different results even when they use the same data and standards. ${ }^{4,5}$ As such, it is critical to "share and compare," then work to resolve differences; ${ }^{5-7}$ this process has been most effectively enabled on a global scale through ClinVar submissions. ClinGen is also a recognized driver project of the Global Alliance for Genomics and Health, which we are working closely with to harmonize standards and best practices for data sharing globally.

It should be noted that most commercial databases, such as the Human Gene Mutation Database, derive content primarily from published literature, which has been documented to be the largest source of incorrect variant classifications. ${ }^{8}$ Also, most commercial databases do not have a good mechanism to allow peer review by the community to validate the content. We support the ClinVar model, which improves over time by allowing ongoing community and expert submissions. We know there is still work to be done to improve the content in ClinVar, and ClinGen is committed to expanding expert panels and discrepancy resolution working groups to support this goal. ClinGen supports transparency in its processes by publishing its methods and providing access to the underlying curation and data sources that support its assertions. We are open to constructive input on our approaches from any source when the goals of the criticism are to improve the quality and comprehensiveness of curated information that is freely accessible to the community.

To attain expert panel (three-star) status within ClinVar, ClinGen requires that groups comprise individuals with 
documented expertise in the genes being curated, and that these groups manage conflicts of interest by avoiding overrepresentation by individual commercial platforms or institutions. ClinGen has not had trouble generating a large number of expert panel committees (http://www.clinicalgen ome.org/working-groups/clinical-domain/) with participation from multiple commercial and academic partners around the world, and these collaborations have been highly effective at aligning approaches to curating variants across many diseases.

We agree with the authors that patients should have the choice of where their data are shared. ClinVar is a variant-centric database, where most submitters simply provide interpretations and de-identified aggregate evidence. Most individual-level data are stored elsewhere based on patient consent. For patient data, we support a federated model similar to that which has been developed for the Matchmaker Exchange (http://www.matchma kerexchange.org). In contrast, coming to consensus on genomic interpretation requires centralization of efforts. It does not, however, require centralization of patient data.

In summary, potential conflicts of interest are always of concern, whether they are alleged towards individuals receiving funding from the $\mathrm{NIH}$ or towards those who are employed by a private company. However, neither I nor any of the other ClinGen principal investigators receive any financial support from the National Center for Biotechnology Information or ClinVar, nor do we contribute any funding directly to ClinVar. ClinGen has always been free to support the sharing of knowledge via any database it chooses. ClinGen's decision to form a close partnership with ClinVar is devoid of conflict of interest as no financial incentives exist in this partnership. We firmly believe that submission to ClinVar is currently the best mechanism to support community exchange of variant interpretations, which facilitates direct application of this knowledge to improve clinical testing, genomic research, and patient care.

\section{DISCLOSURE}

The author is employed by institutions that offer fee-based clinical sequencing, and receives NIH support for the ClinGen program.

\section{Heidi L. Rehm, PhD, FACMG ${ }^{1,2,3}$}

${ }^{1}$ Laboratory for Molecular Medicine, Partners Healthcare Personalized Medicine, Cambridge, Massachusetts, USA; ${ }^{2}$ Department of Pathology, Brigham \& Women's Hospital and Harvard Medical School, Boston, Massachusetts, USA; ${ }^{3}$ The Broad Institute of MIT and Harvard, Cambridge, Massachusetts, USA. Correspondence: Heidi L. Rehm (hrehm@partners.org)

\section{REFERENCES}

1. Baurer P, Karges E, Oprea G, Rolfs A, et al. Unmet needs in human genomic variant interpretation. Genet Med; E-pub ahead of print 26 October, 2017.

2. Rehm HL, et al. A new era in the interpretation of human genomic variation. Genet Med 2017;19:1092-1095.

3. Bale S, Devisscher M, Van Criekinge W, et al. MutaDATABASE: a centralized and standardized DNA variation database. Nat Biotechnol 2011;29:117-118.

4. Amendola LM, Jarvik GP, Leo MC, et al. Performance of ACMG-AMP variant-interpretation guidelines among nine laboratories in the Clinical Sequencing Exploratory Research Consortium. Am J Hum Genet 2016;98:1067-1076.

5. Harrison SM, Dolinsky JS, Knight Johnson AE, et al. Clinical laboratories collaborate to resolve differences in variant interpretations submitted to ClinVar. Genet Med 2017;19:1096-1104.

6. Garber KB, Vincent LM, Alexander JJ, Bean L, Bale S, Hegde M. Reassessment of genomic sequence variation to harmonize interpretation for personalized medicine. Am J Hum Genet 2016;99:1140-1149.

7. Lebo MS, Zakoor KR, Chun K, et al. Data sharing as a national quality improvement program: reporting on BRCA1 and BRCA2 variant-interpretation comparisons through the Canadian Open Genetics Repository (COGR). Genet Med; e-pub ahead of print 20 July 2017.

8. Yang S, Lincoln SE, Kobayashi Y, Nykamp K, Nussbaum RL, Topper S. Sources of discordance among germ-line variant classifications in ClinVar. Genet Med 2017;19:1118-1126.

Advance online publication 2 November 2017. doi:10.1038/gim.2017.179 